\title{
VIRTUAL DESIGN AND CONSTRUCTION: ALIGNING BIM AND LEAN IN PRACTICE
}

\author{
Roar Fosse ${ }^{1}$, Glenn Ballard ${ }^{2}$ and Martin Fischer ${ }^{3}$
}

\begin{abstract}
The construction industry has experienced many inspiring improvements. Especially two movements have introduced innovative design and construction practices: The advancement of lean thinking and tools and maturity in applying BIM and related concepts and technologies. Although Lean and BIM often thrive in the same culture, have many similar properties and strong potential synergies, they are often considered separate initiatives and unfortunately provide only partial benefits when applied on their own. Based on empirical findings of a general contractor, this paper shows how collaborative lean methods and BIM-related technologies can be implemented in an integrated way in practice.
\end{abstract}

Keywords: VDC, BIM, Last Planner, ICE, Big Room, metrics

\section{INTRODUCTION}

The construction industry has for years declined in productivity compared to other industries (Teicholz et al., 2001). One significant factor has been that other industries have proven faster at rethinking processes and applying new technology (Drucker, 2006). However, some improvements are imminent: Lean has emerged as a conceptual approach of optimizing processes, while BIM has rapidly advanced as a transformative information technology, and although conceptually independent, research has found numerous synergies between them (Sack et al., 2010).

As it is sub-optimal to implement potentially efficient technology into poor processes or to support efficient processes by obsolete tools, there is undoubtedly much potential in integrating new technology with efficient work processes. Virtual Design and Construction (VDC), a term defined by the Center for Integrated Facility Engineering (CIFE) at Stanford University, focuses on aligning new technology related to BIM with lean thinking and practices (Kunz and Fischer, 2012; Khanzode et al., 2006).

After years of success with lean and BIM as separate initiatives, several contractors have further benefitted from integrating them in construction projects. The purpose of this paper is to describe how a contractor in Norway has aligned lean practices and BIM technology. The research methods are observations by the lead author, surveys from project participants and two project case studies. Two research questions are posted:

- What are the observed project benefits from effectively combining new ways of collaboration with digital tools?

- What are critical drivers for this change and barriers against it?

\footnotetext{
Regional Manager, Lean Construction, Skanska, Oslo, Norway, +4793444588, roar.fosse@skanska.no Research Director of the Project Production Systems Lab., Univ. of California, Berkeley, CA 94720-1712, USA, +1 415 710-5531, ballard@ce.berkeley.edu

3 Professor, Dept. of Civil and Environmental Engineering, Stanford Univ., Stanford, CA 94305, USA, fischer@stanford.edu
} 


\section{THEORY}

\subsection{Lean and BIM}

The term "lean" was introduced by Krafcik (1988) and applied to construction in 1993. Lean Construction is "the application and adaption of the underlying concepts and principles from the Toyota Production System (TPS) to Construction," (Sacks et al., 2010). Some lean principles are: Understanding value from the client's perspective (including for internal client-supplier relations in production systems and supply chains), reducing waste, improving process stability and transparency, reducing unnecessary buffers and inventory, improving flow with pull-based production, and creating a culture of continuous improvement through employee engagement and the use of metrics and benchmarking (Hines et al., 2011; Sacks et al., 2010; Modig and Åhlström, 2013).

There are several definitions of BIM, and although not conflicting, some refer to BIM as a digital product and others to the process of collaboratively making and utilizing it. BIM is a "digital representation of the physical and functional characteristics of a facility" (Smith, 2007), but also "a verb or adjective phrase to describe tools, processes and technologies that are facilitated by digital machine-readable documentation about the building, its performance, its planning, its construction, and later its operation," (Eastman et al., 2008). Stagg (2012) defines BIM as "a method to describe the project and its spaces, structures, components and materials with their essential information and properties."

Although not dependent on one another (lean practices can be adopted without BIM and BIM can be adopted without lean practices), several researchers claim that notable benefits can be achieved by implementing lean practices and BIM functionality together (Bhatla and Leite, 2010; Khanzode et al., 2006; Khan \& Tzortzopoulos, 2014). By identifying 56 interactions between lean principles and BIM functionality, Sacks et al. (2010) support the hypothesis that the full potential of BIM and lean can only be achieved when their adoption is integrated, and the paper also addresses the need for "a framework that juxtapositions BIM functionalities with lean principles.”

\subsection{Virtual Design and Construction}

VDC can be characterized as an approach of rethinking work processes and organization in construction projects, while supporting these changes by innovative use of new technology. Khanzode et al. (2006) found interactions between VDC and the Lean Project Delivery System, stating that a key benefit is to "simulate the complexities of the construction project delivery, to understand the pitfalls the project teams are likely to encounter, to analyze these pitfalls and address them in a virtual world before any of the construction work ever takes place in the real world.” For instance, 4D-scheduling, which merges a project's BIM and schedule, allows for virtual construction ahead of actual construction, which can reveal issues regarding workflow, safety, logistics, etc.

Fundamentally, a project team cannot produce great results without trustworthy information and reliable workflows. BIM tackles the trustworthiness of information and Lean the reliability of processes. Since the execution of processes both creates and depends on information, one cannot expect to improve the trustworthiness of information without improving process reliability and vice versa. By considering the contributions of BIM and Lean and by combining these with strategies for improved project team collaboration and the formal definition of expected project outcomes, VDC provides a framework to improve productivity and other construction performance objectives. 


\section{PRACTICAL AdOPTION OF VDC}

\subsection{A contractor's VDC-framework}

After years of exploring lean practices and BIM technology, Skanska Norway has recently experienced notable benefits from systematically combining them. For instance, adopting Last Planner to design processes enabled efficient mapping of BIM processes in relation with other ongoing design deliverables (Fosse and Ballard, 2016). Recent development of interactions between collaborative methods and technology, combining lean methods and thinking with BIM, led to a VDC approach inspired by CIFE:

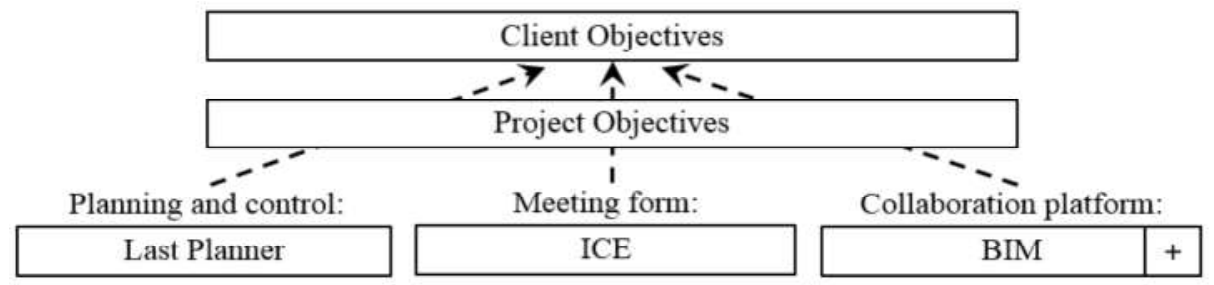

Figure 1: The VDC overview model of Skanska Norway

The lean principle of understanding the end customer's objectives with the project is essential also in VDC, which is evident in Figure 1. The ideal is to provide customers exactly what they need in order to accomplish their purposes, and to do so with minimal waste. Project success criteria, what problem the building is solving for the client, how performance is measured and the objectives of the project team must be aligned.

The Last Planner System (LPS) is an essential collaborative method in any lean project, for mapping both preconstruction and construction activities.

Building Information Modelling (BIM) is essential to VDC. In BIM-mature projects, design coordination is model-based and geometry and object information fulfil the requirements of the Skanska Norway BIM manual. 2D-drawings still exist, but merely as products generated from the BIM. BIM+ refers to advanced BIM applications, such as 4Dscheduling, 5D-cost control, virtual/augmented reality, robotic automation etc.

Integrated Concurrent Engineering (ICE) is a method for multidisciplinary teamwork, originating from NASA's Jet Propulsion Laboratory (Kunz and Fischer, 2012). Whereas traditional meetings often suffer from vague meeting agendas, poor participant preparation and unclear logging of decisions made and follow-up work, ICE sessions counter these challenges with a clear agenda with explicit objectives, active problemsolving, precisely timed tasks and well-prepared, involved participants. ICE utilizes modern collaborative tools and technology, such as BIM on touch screens in a specially designed meeting room, so-called Big Room. An ICE facilitator ensures that the session progresses as planned, and a recorder tracks the results of the session.

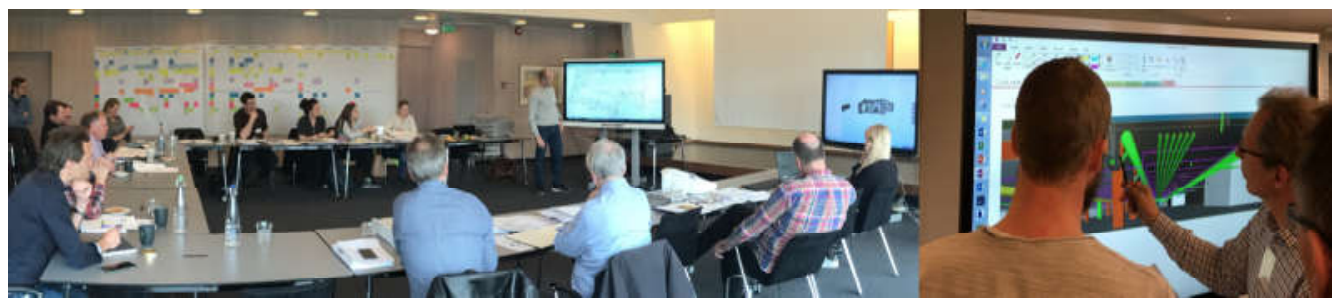

Figure 2: ICE session with Tiedemannsbyen felt A (see case study 2) 


\subsection{Case Study 1: Gjønneshagen}

Gjønneshagen is a residential design-build project of 348MNOK (\$41M), consisting of 181 living units distributed over several residential houses and two apartment blocks. The project's collaborative methods and digitalization started in 2012 with pull-planning of production work of selected phases and areas. As the project went into the third stage, several HSE and quality management processes were digitalized (Autodesk BIM 360 Field), and BIM (Solibri Model Checker) became the main tool for coordinating design and supporting production crews with geometry and information. BIM ambitions continued as a drone laser scan of the construction site was incorporated into the BIM, several materials were procured directly from BIM quantity take-offs and BIM was brought to the workface with on-site BIM stations and tablets (Autodesk 360 Glue).

Last Planner for the design process was implemented as a continuation of the case study in Fosse and Ballard (2015). Shortly after, ICE sessions were implemented as well, after Skanska project participants had received ICE-training from CIFE.

A pilot test of a Last Planner tablet application (Autodesk BIM 360 Plan) made the production schedule accessible and fully updated at all times on all workers' tablets, allowing them to suggest changes, commit to activities and report progress on their own activities directly on their tablets. Most crews were equipped with tablets, and the few without were assisted by Skanska employees who was. With the schedule as a dynamic cloud-based document, the lead planner no longer had to update and distribute the schedule on a weekly basis, but rather spent his time handling the issues within it.

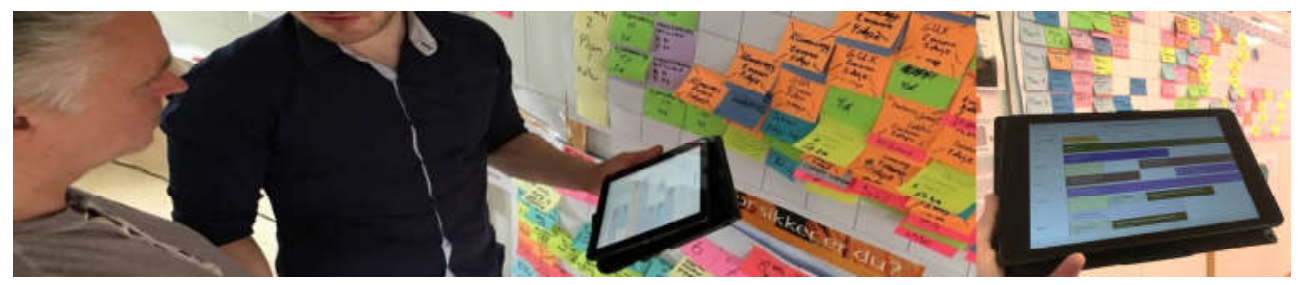

Figure 3: Last Planner System digitalized and tablet-enabled

With only a few months left, the project is on track to completion with good results on a schedule accelerated from 38 to 32 months. The project has served as a learning hub, with project participants moving onto new projects as champions for collaborative methods and digitalization. Several of the innovative practices have been adopted by other projects.

\subsection{Case Study 2: Tiedemannsbyen felt A}

Tiedemannsbyen felt $\mathrm{A}$ is a $458 \mathrm{MNOK}(\$ 54 \mathrm{M})$ residential design-build project consisting of 251 apartments in 5 blocks. The project is a continuation of the practices from the Gjønneshagen project, and VDC implementation started in the spring of 2016. The project was rigged with an on-site Big Room, with digital touch screens and wall space for Last Planner plans. Although primarily for ICE sessions, the room has been used for several purposes within and between design, procurement and production teams. Daily BIM routines, such as model updates, clash detections and quantity take-offs, have been performed by the project team rather than a BIM coordinator from the central Skanska BIM department. This has ensured several benefits: Firstly, the project's funds for a BIM coordinator are allocated for technical support and further BIM development rather than performing daily routines. Secondly, with increased ownership and knowledge of BIM methods, project team members have replaced or supported existing tasks with BIM 
functionality. Thirdly, the quality of BIM deliverables has increased, as the person performing the tasks has more knowledge of the significant issues in the project as well as more industry experience than a BIM coordinator. Metrics for Last Planner (PPC, root cause trends, task completion rate, reliability per trade), BIM clash trends per area according to the LOD-plan and ICE session evaluations (session efficiency, team preparations and involvement) have been continuously tracked and put on the wall in the Big Room, ensuring transparency and control of the project team's performance.

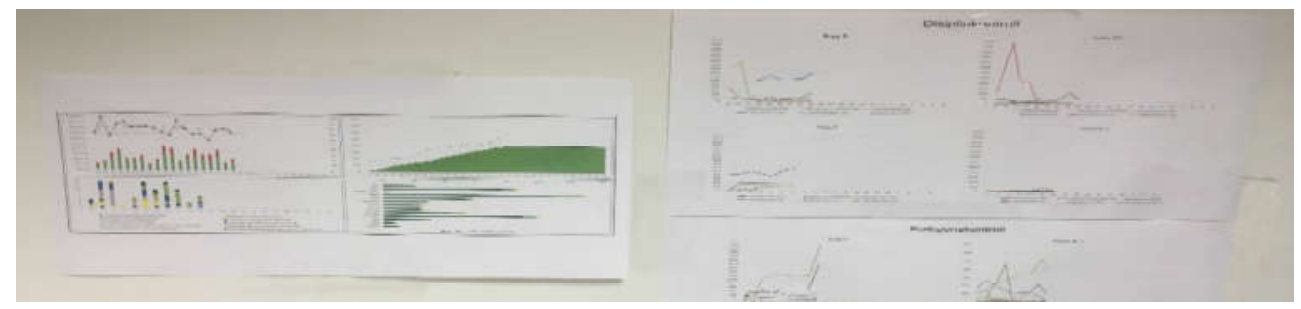

Figure 4: Last Planner and BIM metrics on the Big Room wall

A Level of Development (LOD) system, which is a status system of maturity of different objects and areas in the BIM, has been mapped on the wall with LOD-milestones as part of the Last Planner design plan. The LOD-milestones were pulled from the needs of onsite crews for a constructible BIM in time for production. The BIM has been available to all on-site crews through tablets with BIM 360 Glue, as well as in the office with Solibri. Skanska crews have also had a BIM station in a crew container, which has been especially useful for the crew that assembles rebar directly from the BIM rather than drawings. The BIM has been accessible on touch screens in the Big Room in most meetings, both for design teams and production crews. Especially in the Last Planner sessions, it has been used frequently as a discussion basis.

Everyone on the project has been offered BIM training, both for applications in the office and on-site. Every trade has also been trained in Last Planner as part of their participation in pull-planning sessions for either design processes or production work.

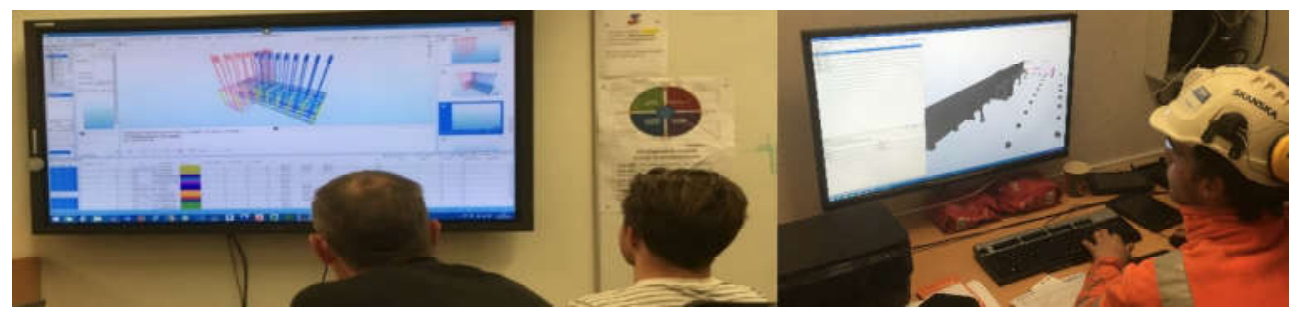

Figure 5: BIM on touch screen in project Big Room and in on-site BIM container

Although still early in terms of measuring project performance, some benefits have been observed from VDC practices. The design schedule has been reduced, allowing the senior design manager to leave the project 6 months earlier than initially planned. Members of the design team have claimed that there is a noticeable difference between the reliability and efficiency of this project compared to other projects they work with, and one major design firm has requested to adapt the project's methods to their own company. The client representative claims that the VDC methods have greatly increased the team's understanding between project team and client objectives, and has also requested training to initiate similar processes on other projects. 


\section{DISCUSSION}

The two research questions posed in the introduction were:

- What are observed project benefits from effectively combining new ways of collaboration with digital tools?

- What are critical drivers for this change and barriers against it?

In 20 months, the first author of this paper has facilitated 59 ICE sessions, 55 pull-planning sessions and 68 plan checks for 28 construction projects, with about half of them having a holistic VDC approach as described in this paper. Although challenging to quantify the effects of VDC practices, some benefits are clearly observed. One Skanska design manager claims that VDC practices reduced his project's design phase by at least 4 months (27\%), mostly by reducing waiting time and misunderstandings. In addition to measuring PPC on schedules and BIM clashes per area, ICE sessions are evaluated with anonymous survey asking the team how prepared they felt, how well they feel they participated and how efficient they feel the meeting was. The average score from a number of ICE sessions across the Oslo region indicate that ICE is beneficial:

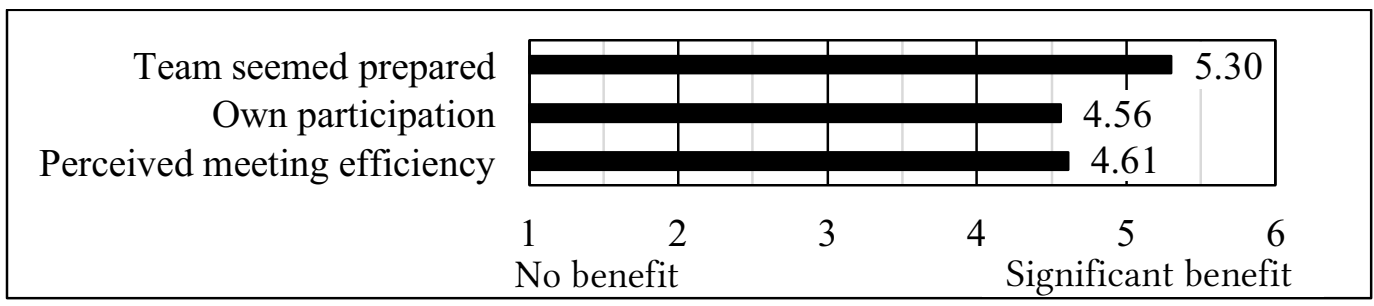

Figure 6: ICE evaluations (average team score from 5 sessions for first question and average team score from 26 ICE sessions for the other two).

A survey asked 143 participants from Skanska projects across Norway how they feel about pull-planning sessions compared to previous planning techniques, and results indicate that they feel pull-planning increased the quality of commitments within the team, resulted in a more realistic plan and a greater understanding of the planned phase:

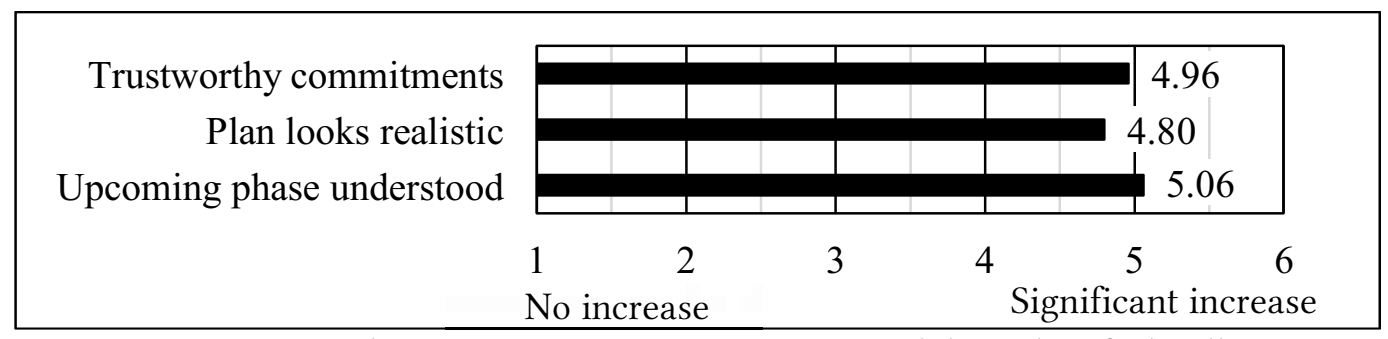

Figure 7: Survey asking 143 project participants to rank how they feel pull-

planning sessions increased some key factors as opposed to traditional planning.

A key motivation for implementing VDC was to better coordinate efforts related to Lean principles and methods, especially the Last Planner System, BIM, ICE and metrics. According to the head of the BIM department of Skanska Norway, BIM is now better integrated in all project phases, and the BIM process is better understood by project teams through mapping it on the wall in the early design-focused Last Planner process. In return, the model is a central source of information in pull-planning of production, imperative to on-site production information with tablets and stationary BIM units, as well as being the 
main discussion basis in ICE meetings. The plan on the wall functions as a canvas for visualizing consequences and opportunities of issues the team works on in the ICE sessions. Metrics of all three methods, such as ICE efficiency, Last Planner PPC and root cause trends, as well as BIM clash trends, have ensured control of team performance.

A lean culture is more evident in VDC projects, where project participants see each other in client-supplier relations, focus on delivering value to each other, measure and control team performance and continuously improve processes, tools, and methods.

Regarding drivers for and barriers to change, four elements have been crucial when evaluating the Skanska Norway VDC initiative recently: Knowledge of the methods and how to implement them, an innovative lean culture on a project level, proper training of key personnel and leadership that understands and promotes the approach. The first three factors have been championed by the regional lean manager (first author of this paper), alongside a continuously growing movement of project participants across the Oslo region who have participated in promoting and developing the VDC methods. Regional and national leadership has been positively outspoken about VDC as a collaboration and digitalization approach, as indicated by one Oslo district manager: "VDC increases our ability to realize lean principles, like involving people in seeing the solutions together, and use BIM and other digital tools in a sound way."

No noteworthy barriers against change have been encountered, but some potential threats have been identified: Lack of funding for necessary tools and training, unclear goals and implementation plans and insufficient knowledge of strengths and weaknesses of methods. Funding has been solved by projects, clear goals has been achieved by putting the VDC effort into regional and national strategies, implementation plans have been developed collaboratively with project participants and substantial testing of equipment, software and methods have been done in controlled pilot tests before scaling up. Furthermore, a common barrier against change is that the time and effort necessary to achieve positive results is underestimated. This has not been observed in the VDC effort, perhaps due to the contractors years of experience implementing lean and BIM.

It has been important to "pull" innovation bottom-up, rather than "push" it top-down: VDC practices are continuously improved on a project level, and project needs regarding implementation or knowledge sharing is accommodated by management. Unfortunately, change initiatives are often implemented top-down, with new methods and tools pushed from a managerial level upon a workforce who does not want the change or see the need for new practices. Another barrier to change is to spend time and focus on working against those who do not want change. A central aspect to the VDC implementation has been to fully commit to those who want the change and not against those who do not. The belief is that those who are against the change will see the results and join the movement.

\section{CONCLUSIONS}

After years of exploring how to align digital tools and collaborative methods, Skanska Norway has recently benefitted from the terminology and understanding of Virtual Design and Construction. Several elements of lean construction are prevalent in the approach, and especially the combination of Last Planner, BIM and ICE sessions has been beneficial, as described in two case studies. Key drivers for positive change has been that the development of VDC practices are done on a project-level, with sufficient training provided and that leadership promotes the practices. No notable barriers have been encountered, but potential threats include lack of funding, unclear goals and implementation plans and underestimated time and effort needed for positive results. 
Although difficult to evaluate the impact of VDC practices directly on project performance, there are several indications that VDC practices have had positive impact.

\section{ACKNOWLEDGMENTS}

The case project teams have shown a tremendous interest and willingness to adopt new practices, as well as contributing with research data in this paper. The BIM department, IT department and Digital Construction department have been crucial to the success of the methods described in this paper through their formidable efforts to collectively challenge common practice and develop innovative workflows to enable new technology. The Oslo regional management and executive directors of Skanska Norway must also be acknowledged for their immense support and encouragement of the VDC initiative.

\section{REFERENCES}

Alves, T., Milberg, C., and Walsh, K. D. (2012). "Exploring lean construction practice, research, and education." Engineering, Construction and Architectural Management, Vol. 19 Iss: 5

Diekmann, J. E., Krewedl, M., Balonick, J., Stewart, T. \& Won, S. (2004). "Application of lean manufacturing principles to construction." CII Report, Uni. of Texas, Austin

Drucker, P. (2006). "Innovation and Entrepreneurship, Practice and Principles." Harper Collins, reprint edition, May 9th 2006

Fosse, R. and Ballard, G. (2016). "Lean Design Management in Practice with the Last Planner System. " Proceedings IGLC 24, Boston, MA, USA

Hines, P., Found, P., Griffiths, G. and Harrison R. (2011). "Staying Lean: Thriving, not just surviving. "Productivity Press

Khan, S. and Tzortzopoulos, P. (2014). "Effects of the interactions between LPS and BIM in two construction projects. "Proceedings IGLC 22, Oslo, Norway

Khanzode, A., Fischer, M., Reed, M. and Ballard, G. (2006). "A guide to applying the principles of Virtual Design \& Construction (VDC) to the Lean Project Delivery Process. "CIFE Working Paper \#093, Stanford, CA

Koskela, L. (1992). "Application of the new production philosophy to construction." Technical Report No. 72, Center for Integrated Facility Engineering, Stanford, CA

Krafcik, J. F. (1988). "Triumph of the lean production system." MIT Sloan Management Review 30.1: 41

Kunz, J. and Fischer, M. (2012). "Virtual Design cases \& studies." Working Paper \#097, Center for Integrated Facility Engineering, Stanford, CA

Modig, N. and Åhlström, P. (2012). "This Is Lean: Solving the Efficiency Paradox." Rheologica Publishing, Sweden.

Sacks R., Koskela, L., Dave, B. and Owen, R., (2010). "Interaction of Lean and Building Information Modelling in Construction." Journal of Construction Engineering and Management, ASCE, 136(9), p. 968-980

Teicholz, P., Goodrum, P. M. and Haas, C. T., (2001). "U.S. Construction Labor Productivity Trends, 1970-1998." Journal of Construction Engineering and Management, Sept/Oct 2001, p. 427-429 